\title{
Structural Characterization of Intact Proteins Is Enhanced by Prevalent Fragmentation Pathways Rarely Observed for Peptides
}

\author{
Jennifer S. Cobb, ${ }^{\text {a }}$ Michael L. Easterling, ${ }^{b}$ and Jeffrey N. Agar ${ }^{a}$ \\ a Department of Chemistry and Volen Center for Complex Systems, Brandeis University, Waltham, \\ Massachusetts, USA \\ ${ }^{\mathrm{b}}$ Bruker Daltonics Inc., Billerica, Massachusetts, USA
}

While collisionally activated dissociation (CAD) pathways for peptides are well characterized, those of intact proteins are not. We systematically assigned CAD product ions of ubiquitin, myoglobin, and bovine serum albumin generated using high-yield, in-source fragmentation. Assignment of $>98 \%$ of hundreds of product ions implies that the fragmentation pathways described are representative of the major pathways. Protein dissociation mechanisms were found to be modulated by both source declustering potential and precursor ion charge state. Like peptides, higher charge states of proteins fragmented at lower energies next to Pro, via mobile protons, while lower charge states fragmented at higher energies after Asp and Glu, via localized protons. Unlike peptides, however, predominant fragmentation channels of proteins occurred at intermediate charge states via non-canonical mechanisms and produced extensive internal fragmentation. The non-canonical mechanisms include prominent cleavages Cterminal to Pro and Asn, and N-terminal to Ile, Leu, and Ser; these cleavages, along with internal fragments, led to a $45 \%$ increase in sequence coverage, improving the specificity of top-down protein identification. Three applications take advantage of the different mechanisms of protein fragmentation. First, modulation of declustering potential selectively fragments different charge states, allowing the source region to be used as the first stage of a low-resolution tandem mass spectrometer, facilitating pseudo-MS ${ }^{3}$ of product ions with known parent charge states. Second, development and integration of automated modulation of ion funnel declustering potential allows users access to a particular fragmentation mechanism, yielding facile cleavage on a liquid chromatography timescale. Third, augmentation of a top-down search engine improved protein characterization. (J Am Soc Mass Spectrom 2010, 21, 949-959) (c) 2010 American Society for Mass Spectrometry

$\mathrm{M}$ ass spectrometry (MS) is a useful tool for characterizing the composition and structure of molecules. The soft ionization methods of electrospray ionization (ESI) [1] and matrix-assisted laser desorption/ionization (MALDI) [2] extended the mass range of MS to include intact proteins, and enabled "top-down" mass spectrometry approaches. There are two fundamental differences between the so-called top-down and the traditional bottom-up approaches (bottom-up uses proteolytic peptides of $\leq 3000$ Da) [3]. The first is that top-down methods can provide the mass of the protein and the second is that top-down proteomics methods rely exclusively upon fragments generated in the gas phase, whereas bottom-up methods employ an additional chemical or enzymatic digestion step [3]. Limitations of top-down methods are the upper mass limit (currently at $669 \mathrm{kDa}$ [4] for protein

Address reprint requests to Dr. J. N. Agar, Department of Chemistry and Volen Center for Complex Systems, Brandeis University, 415 South Street, MS 015, Waltham, MA 02454, USA. E-mail: agar@brandeis.edu identification) and the efficiency, and understanding of the mechanism of, gas-phase protein fragmentation.

Numerous surface-induced dissociation and collisionally activated dissociation (CAD) studies [5-12] characterized peptide fragmentation mechanisms [5], including the comprehensive characterization of fragmentation trends by systematic, large-scale studies [13-15]. From these studies arose the "mobile proton model" (and the recent "pathways in competition" model), which state that fragmentation requires the collisionally activated transfer of a proton from a basic site to a less basic site. The positions of amino acids with high structural rigidity and high gas-phase basicity (proline, arginine, histidine, lysine, and the protein $\mathrm{N}$-terminus) thus greatly influence fragmentation [6, 9, 16, 17]. If mobile protons are not available or if sufficient energy is present, fragmentation proceeds using a localized (and relatively immobile) proton from an acidic residue, resulting in cleavage C-terminal to Asp or Glu $[5,18]$.

Because peptides are essentially shorter versions of proteins, one could question whether anything additional 
can be learned from studies of protein CAD. However, fundamental differences between proteins and peptides exist [19], including size, representative terminal residues, and the strikingly low sequence coverage of intact protein CAD. For example, the C-terminal Arg and Lys of tryptic peptides play important roles in peptide CAD mechanisms, and when combined with the low charge states of peptides, bias against the observation of b-ions. With proteins, however, the C-terminal residue can be any amino acid, more charges are present, and as a result a larger fraction of b-ions should be observed. Another difference is that the $\mathrm{N}$-terminus of most tryptic peptides is a free amine that possesses an additional proton compared with a modified $\mathrm{N}$-terminus. While this proton can be sequestered at the N-terminus [20], the paucity of b-ions in peptide CAD is consistent with its frequent mobilization. Half of yeast and the majority of mammalian proteins [21], however, have acetylated N-termini, which have lower gas-phase basicities than intact amino termini and are even less likely to sequester a proton [6, 20]. Moreover, proteins have a broader array of conformational structures and charge densities. Clearly, the range of possible fragmentation mechanisms available to proteins is higher than for peptides.

Methods of protein fragmentation include CAD in a multipole [22], sustained off-resonance irradiation (SORI)CAD [23], infrared multiphoton dissociation (IRMPD) [24], ECD [25], electron-transfer dissociation (ETD) [26], and the in-source fragmentation methods of prefolding dissociation (PFD) [27], nozzle-skimmer dissociation (NSD) [28], and, as discussed here, funnel-skimmer dissociation [4]. One drawback of NSD is the tendency to lose ions through scattering before they enter the relatively narrow skimmer aperture [29]. Funnel-skimmer dissociation of ions, on the other hand, occurs in the relatively wide ion funnel inter-stage region between the first [30] and second funnel (see Figure 1) providing higher transfer efficiency for large, multiply charged fragment ions [29-31]. Generally speaking, methods for intact protein fragmentation provide far less sequence coverage than bottom-up methods. For example, electron capture dissociation (ECD) efficiency is theoretically limited to $33 \%$ [32] with typical experimental efficiencies of less than $10 \%$.

As a result of the low fragmentation efficiency of ECD, high throughput top-down studies have resorted to CAD for protein identification [33, 34]. Intact protein fragmentation mechanistic studies have been spearheaded by groups including McLuckey, Reid, McLafferty, and Clemmer [35-41]. However, important questions still remain, for example, why intact protein CAD generates predominantly $\mathrm{N}$ - and C-terminal fragments and low yields, and whether proteins and peptides have dissimilar dissociation mechanisms [42]. For example, many intact protein fragment ions are not well understood and have been characterized as "relatively nonspecific" [37]. A better understanding of the mechanisms of intact protein fragmentation would improve our fundamental understanding of gas-phase ionmolecule chemistry and could be applied for improving experimental conditions and design, interpreting results, and optimizing search engine specificity of protein identification algorithms [4].

The goals of this study are to further characterize the mechanisms of intact protein CAD, and funnelskimmer dissociation in particular. Here, we explore energy and charge-state dependence of the CAD of intact proteins, and characterize qualitative differences between the fragmentation channels of proteins and peptides. We develop new electronics that when combined with optimized instrument parameters and mechanism-minded database searching, allow for identifying and characterizing proteins on a LC timescale, a feat that cannot currently be accomplished using ECD. Continued expansion of top-down efforts $[39,43,44]$ and persistent interest and discoveries related to peptide fragmentation [45-48] make this study generally applicable to the broad discipline of chemistry and related sciences.

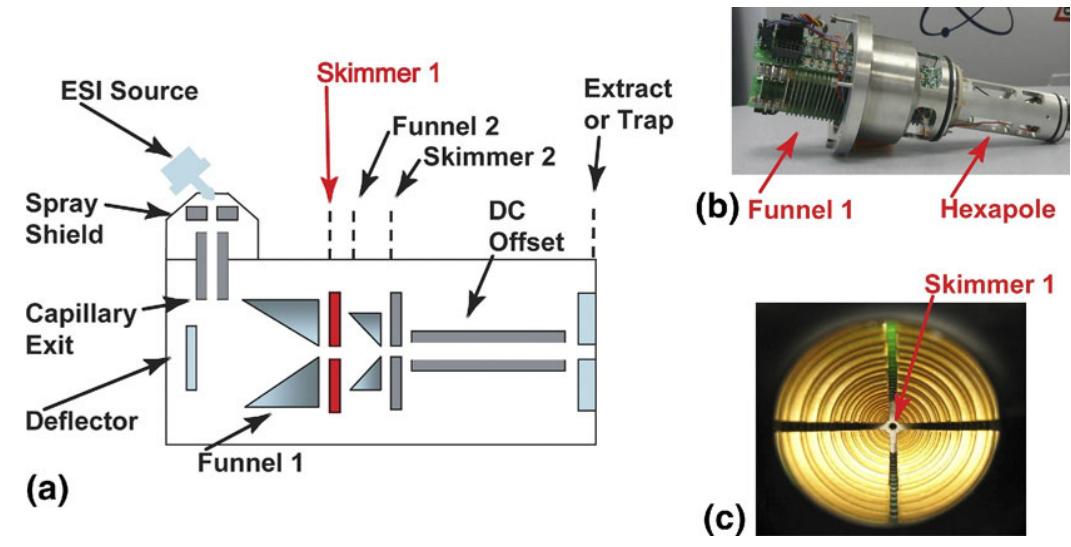

Figure 1. Depiction of the FT-ICR mass spectrometer source schematic. (a) FT-ICR Apollo II source diagram, where potentials help guide the ions further into the instrument. Increased declustering potential (ion optic skimmer 1) caused fragmentation. (b) Picture of a dual ion funnel with attached hexapole from the instrument, labeled for orientation to compare to (a). (c) Looking down ion funnel 1 at the decreasing inner diameter of the disk electrodes, one can see the labeled optic skimmer 1. 


\section{Experimental}

\section{Sample Preparation}

All solvents (HPLC grade), chemicals, and proteins were purchased from Sigma-Aldrich (St. Louis, MO, USA) unless otherwise stated. Bovine ubiquitin (8.6 $\mathrm{kDa}$ ) (Boston Biochem, Cambridge, MA, USA) was desalted using an Amicon Ultra-4 centrifugal filter device (Millipore, Billerica, MA, USA) with a 5000 nominal molecular weight cutoff. The filters were used as directed with the exception of $10 \mathrm{mM}$ ammonium bicarbonate; $\mathrm{pH} 7.4$ was added to the first wash of the protein. Water was used for the remainder of three washes. Equine myoglobin $(16.9 \mathrm{kDa})$ was used without further purification. Bovine serum albumin (BSA, 66.4 $\mathrm{kDa}$ ) was prepared in $100 \mu \mathrm{M}$ solution and the disulfide bonds were reduced with $50 \mu \mathrm{M}$ dithiothreitol (DTT) (Fisher Scientific, Pittsburgh, PA, USA) at $60{ }^{\circ} \mathrm{C}$ for 15 min. The concentrations of ubiquitin, myoglobin, and BSA prepared for mass spectrometry analysis in 50\% water: $50 \%$ acetonitrile solution containing $0.1 \%$ formic acid were $0.5,0.5$, and $1 \mu \mathrm{M}$, respectively.

\section{Fourier Transform-Ion Cyclotron Resonance (FT-ICR) Mass Spectrometry Analyses}

All experiments were performed using a Bruker Daltonics Inc. Apex Qe-94 equipped with a dual stage ion funnel, quadrupole 9.4 Tesla FT-ICR mass spectrometer (Billerica, MA, USA). Following initial optimization of ion source conditions, external calibration in the $\mathrm{m} / \mathrm{z}$ domain was performed with electrospray tuning mix (Agilent, Santa Clara, CA, USA) using peaks at $m / z$ 622, 922, 1522, and 2122. Samples were directly infused into the mass spectrometer via an Apollo II electrospray ion source (Bruker Daltonics Inc.) at a flow rate of $110 \mu \mathrm{L} / \mathrm{h}$ maintained with a syringe pump (Cole Parmer, Vernon Hills, IL, USA). The experimental setup for the source is illustrated in Figure 1a. The inlet capillary voltage was set at $-4.4 \mathrm{kV}$, and the capillary was heated to four temperatures $\left(100,150,200\right.$, and $\left.240^{\circ} \mathrm{C}\right)$ to determine the optimal capillary temperature to aid in fragmentation. Biasing the outlet capillary to $310 \mathrm{~V}$ helped guide desolvated positive ions into the funnel-skimmer region. Ions moved from a source hexapole ion guide (accumulated $0.05 \mathrm{~s}$ ) to the quadrupole region before transmission into a second hexapole. Ions accumulated in the hexapole ion guide for $1 \mathrm{~s}$ then were transferred to the analyzer cylindrical ICR cell via electrostatic ion optics. Ions were excited using a frequency-sweep (chirp) waveform, and acquisitions were recorded using $512 \mathrm{k}$ data points in broadband detection mode.

\section{Funnel-Skimmer Dissociation}

Fragmentation occurred by collision of ions with background gas (nitrogen), where the pressure in this region was $\sim 3$ mTorr $[4,49]$. Note that the declustering potential was predominantly in the ion optic skimmer 1 , therefore when this voltage was increased (as shown in Figure 1), fragmentation of proteins could be induced. Skimmer 1 is different than the conventional use of a molecular beam-like skimmer in that for funnel-skimmer dissociation, this voltage increase caused the conductance limit and pressure differential to increase while decreasing from ambient pressure at $\sim 3$ Torr down to mTorr.

Details of typical positive ion mode tuning voltages for the source have been described recently [4]. Instrument parameters shown in Figure 1a such as capillary temperature, ion funnels 1 and 2, skimmers 1 and 2, deflector, capillary exit- and extract/trap-voltages, time of flight, and analyzer (sidekick, excitation amplitude) were cursorily set once an optimal signal was obtained by looking at their effects on signal intensity and fragmentation. Results were visually evaluated for changes in fragment ion intensity. These voltages in the source funnel-skimmer region, apart from the skimmer 1 declustering potential, were set to achieve optimal spectral intensity. As shown in Figure S1, (in Supplemental Information, which can be found in the electronic version of this article), by setting the declustering potential at $40 \mathrm{~V}$, the basic work flow for funnelskimmer dissociation fragmentation includes intact protein molecular weight determination. This voltage allowed good sensitivity in ion signal transmission with reduction of solvent adducts, while also allowing little to no fragmentation of the precursor protein that is useful for protein identification. Funnel-skimmer dissociation fragments were produced by increasing declustering potential voltage to $60-140 \mathrm{~V}$, with fragmentation tested by incremental increases of $10 \mathrm{~V}$.

Source and instrument parameters for funnel-skimmer dissociation experiments were the same for all proteins. Nonetheless, differences worth noting included the following parameters: (1) ubiquitin and BSA: mass range $=m / z 100-4000$, time of flight delay $=1.5 \mathrm{~ms}$, quadrupole 1 mass $=m / z$ 400, quadrupole pressure $=$ $2.7 \times 10^{-6}$ torr; (2) myoglobin: mass range $=\mathrm{m} / \mathrm{z}$ 200-3000, time of flight delay $=2 \mathrm{~ms}, \mathrm{Q} 1$ mass $=m / z$ 600 , quadrupole pressure $=2.25 \times 10^{-6}$ torr. Experiments were conducted in chromatography mode. Funnelskimmer dissociation resulted in CAD-type fragmentation with cleavage at the amide bond yielding $b$ - and $y$ ions (and some neutral losses), retaining the $\mathrm{N}$ - and C-terminus respectively, with the fragmentation nomenclature summarized by Roepstorff and Fohlman [50], and Biemann [51].

\section{Automation of Funnel-Skimmer Dissociation}

Automatic modulation of the optimized MS and CAD parameters was accomplished by using a TTL pulse clocked after every scan to modulate the voltage of the declustering potential (skimmer 1) from low to high values and back. This pulse could be generated in two ways: (1) pulse program integrated into APEX Control 
software (Bruker Daltonics) synchronized with the TTL switching, running short $(\sim 200 \mathrm{~ms})$ hexapole fill times for the MS scans and long $(\sim 1-60 \mathrm{~s})$ fill times for the CAD scans; or 2) pulse generator (DG535, Digital Delay Pulse Generator; Stanford Research Systems, Sunnyvale, CA, USA) set to create the chosen pulse frequency (e.g., $0.333 \mathrm{~Hz}$ ). Experiments described here were performed with the integrated pulse program. Figure S2 shows the schematic for the construction of the electronics needed to create the TTL pulse producing MS or $\mathrm{MS}^{2}$. For an automated pulse program using the software, the TTL dual D-type positive edge triggered flip-flop (SN74LS74AN; TX Instruments, Waltham, MA, USA) box ( $10 \mathrm{k} \Omega$ resistor, $0.1 \mu \mathrm{F}$ capacitor, and powered with $9 \mathrm{~V}$ battery) was wired via BNC cables into the back of the Acquisition Control System power console to the specific output to be called to in the pulse program. The resulting TTL pulse fed into a $150 \mathrm{~V}$ power supply with the output looped to another BNC connection, wired to the skimmer 1 voltage wire on the source of the instrument. This connection was split so that readout could be monitored with a voltmeter (Wavetek Meterman, Everett, WA, USA). Low and high voltages were set using potentiometers (Bourns, Riverside, CA, USA).

\section{Data Analysis and Database Searching}

Raw data were deconvoluted and the monoisotopic mass was found using the Sophisticated Numerical Annotation Procedure (SNAP) II algorithm in DataAnalysis V. 3.4 (Bruker Daltonics) [52]. $[\mathrm{M}+\mathrm{H}]^{+}$ masses with intensities were searched using the topdown database search engine, Mascot-TD (Matrix Science Inc., Boston, MA, USA) [4, 53]. Search specifications included "none" as the enzymatic digestion and amino (N)-terminal acetylation as a variable modification, searched in the MSDB database. Default error tolerances for peptide mass (or MS ${ }^{1}$ were set to $\pm 0.1 \%$ for ubiquitin and BSA or $\pm 1 \%$ for myoglobin to take into account the potential of electrospray adducts to the intact protein mass or the potential for the partial reduction of disulfide bonds. Fragment mass tolerances (or funnel-skimmer dissociation-MS ${ }^{2}$ were set to 0.010.3 Da depending on the search performed. These MS ${ }^{1}$ and $\mathrm{MS}^{2}$ tolerances are larger than they need to be considering the accuracy of the FT-ICR instrument used but were chosen to be representative of high throughput LC/MS-based studies where ion abundances vary greatly, and because Mascot-TD currently does not allow for ppm-based $\mathrm{MS}^{2}$ tolerances. Intact protein $[\mathrm{M}+\mathrm{H}]^{+}$masses were used as precursor masses for the Mascot-TD searches, except in the case of BSA where 60 $\mathrm{kDa}$ was used, $60 \mathrm{kDa}$ being the upper limit for the precursor ion mass at the time when searches were performed (currently $110 \mathrm{kDa}$ ) [54]. Additionally, internal fragment searches incorporated new user-defined instrument definitions into Mascot-TD, as a large proportion of unmatched ions existed in the raw data. One definition added a-type fragments and ya- and yb internal fragments up to $30 \mathrm{kDa}$ in size to be searched, in addition to the usual b- and y-fragment ions and their water and ammonia loses. The second definition added only yb-internal fragments up to $30 \mathrm{kDa}$, in addition to the usual $b$ - and $y$ fragment ions.

\section{Mechanistic Considerations for Improved Protein Identification}

To determine the best experimental conditions for protein identification, a relationship between Mascot-TD score and declustering potential was explored. The Mascot-TD probability based algorithm assigns a MOWSE score to matches and decides the significance of the score based on sequence homology [4,53]. The combined fragmentation and intact protein $[\mathrm{M}+\mathrm{H}]^{+}$ masses and intensity were searched in the Mascot-TD database. The higher the number of fragments identified out of the total number submitted (including loss of water and ammonia, etc) constitutes the maximum score. Illustrated in Figure S3 is the bimodal distribution with two local maxima at 80 and $120 \mathrm{~V}$ for ubiquitin, consistent for all temperatures examined. We tested whether the multiple distributions, observed for all proteins studied (Figures S4 and S5), suggested if certain energy thresholds existed where more fragmentation was observed (in terms of higher scores) or if perhaps certain fragmentation channels were not detected by Mascot-TD.

\section{OriginPro Data Analysis}

OriginPro 8 (OriginLab Corp., Northampton, MA, USA) software was used for extended data analysis and graphing ability. The plotted data symbol (which signified the mass of the fragment at the declustering voltage of interest) was modified so that the color could be mapped based on the intensity of the fragment. The minimum and maximum intensity levels were set to encompass fragments of all declustering voltages used experimentally. The color mapping was accomplished by plotting the intensity on a log scale with the number of color intensity levels set the same for all plots (i.e., 1000). Low intensity was displayed as blue and high intensity was displayed as red, with limited mixing as a gradient transition from one color to another. The unnormalized intensity was used (instead of total ion chromatogram or base peak chromatogram normalization) since the standard deviation of the intensity monitored over the entire experiment was within $8.2 \%$.

\section{Spectral Validity Data Analysis and Internal Fragmentation}

Funnel-skimmer dissociation peaks for each declustering potential were manually verified to check for charge-state assignment and validity of the masses. The 
identification of each peak was assigned when possible, whether the peak was an a-, b-, or y-ion, a neutral loss, or even an internal fragment. Because of the high mass accuracy of FT-ICR MS masses, redundant internal fragment masses were assigned to a specific fragment when possible. When multiple possibilities of internal fragments resulted, the error in part-per-million (ppm) was calculated. As long as the root-mean-square (RMS) error was reasonable within the Mascot-TD search parameters used, the fragment was identified (e.g., internally calibrated ubiquitin had a RMS error $<10$ ppm; see Table S1 for details).

\section{Results and Discussion}

As a result of the low fragmentation efficiency of ECD, high throughput top-down studies have resorted to CAD for protein identification $[33,34]$. Whereas CAD of peptides often yields numerous assignable product ions and remains the method of choice for proteomics studies, CAD of intact proteins yields relatively fewer assignable products and therefore less comprehensive structural characterization [42]. A better understanding of the mechanisms of intact protein CAD should improve matters. Promising results, including higher fragmentation efficiency than traditional CAD in a collision cell, and the identification of a $669 \mathrm{kDa}$ protein [4], led us to characterize the mechanisms of in-source (funnelskimmer) CAD and to optimize it for high-throughput studies. Our results are expected to be applicable to CAD in general, for example to ion-trap and hexapole CAD. Here we characterize the mechanism of CAD of proteins as a function of charge state, source declustering potential, and capillary temperature (described in the Experimental section). We identify "sweet spots" in declustering potential and protein charge state that arise from mechanisms not often observed in peptides and that result in substantially improved fragmentation efficiency, sequence coverage, and structural characterization. These "sweet spots," which were once believed to result from unselective cleavage [37], are shown to result from internal fragments of multiple mechanistic origins.

Taking advantage of these mechanisms, we demonstrate a general method for using the source region as a low-resolution mass spectrometer, and provide experimental guidelines for tailoring protein fragmentation channels (e.g., cleavage at proline versus aspartic acid). We then construct electronics that can alternate between the ideal declustering potentials for determining intact protein mass and protein fragmentation. Finally, we demonstrate that sequence coverage can be significantly improved and confidence in protein identification increased by taking internal fragments into consideration using an automated, commercially available search engine (the Mascot-TD search database [4, 53]).

\section{Similarities in Collisionally Activated Dissociation Mechanisms Observed for Proteins and Peptides}

Three proteins were chosen for this study that were within a molecular weight range that contains $80 \%$ of human proteins (8.6 to $66.4 \mathrm{kDa}$ ) [4]. We attained intact protein molecular weights at a declustering potential of $40 \mathrm{~V}$, which afforded good sensitivity, no solvent adducts, and little detectable fragmentation. Upon further increasing this potential from 60 to $140 \mathrm{~V}$ dissociation resulted. By virtue of having comprehensively assigned $>98 \%$ of the fragment ions for ubiquitin, we assert that no prevalent fragmentation channel went unassigned. The conclusions and generalizations reached with ubiquitin were then verified for myoglobin and BSA (see Figures S7 and S8).

Figure 2 illustrates that the higher the charge state, the lower the declustering potential threshold for dissociation [55], consistent with previous in-source and collision-cell CAD studies [28, 56-61]. This affords the indirect selection of the transmitted and dissociated
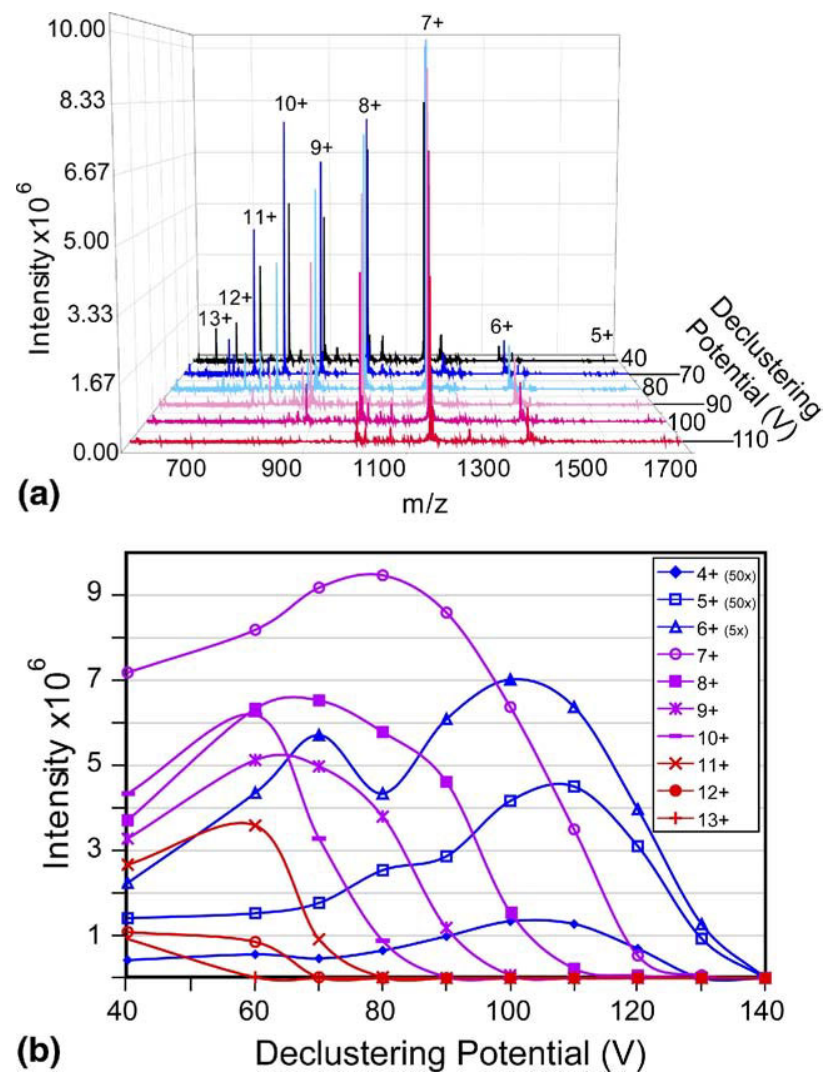

Figure 2. (a) Higher charge states dissociate at relatively lower declustering potential. Three-dimensional plot of ESI mass spectra of ubiquitin $[\mathrm{M}+13 \mathrm{H}]^{13+}$ to $[\mathrm{M}+5 \mathrm{H}]^{5+}$ charge states. Increased declustering potential first caused decreased abundance of the more highly charged molecular ions and a shift towards transmission of lower charged molecular ions for ubiquitin. (b) Charge state selection based on declustering potential. Intensity of each ubiquitin parent ion charge state $\left([\mathrm{M}+13 \mathrm{H}]^{13+}\right.$ to $\left.[\mathrm{M}+4 \mathrm{H}]^{4+}\right)$ at specific declustering potentials. Note: $4+$ and $5+$ charge states were scaled by $50 \times$ their actual intensities, and $6+$ was scaled by $5 \times$ to visualize the declustering potential effect for low intensity charge states. 
charge states by using different declustering potentials. The dissociation of higher charge states tended to give rise to cleavage at the N-terminus of Pro at low declustering potential, whereas lower charge states gave rise to C-terminal Asp/Glu cleavages (Figure 2) at higher declustering potential, consistent with previous protein studies [37, 62-69]. Such fragmentation was extensive (Figure 3). For example, dissociation of ubiquitin occurred at low declustering potentials preceding (Nterminal to) all proline residues, and at high declustering potentials following all aspartic and glutamic acid residues (Figure S6; Figures S7 and S8 for myoglobin and BSA details, respectively). Based upon previous studies of peptides, other likely cleavages included Ile/Leu/Val-Xaa, which we also observed. Facile dissociation of more highly charged states has been attributed to greater numbers of mobile protons, and to increased lability due to destabilization [70] and more unfolded states [71], both of which result from the Coulombic repulsion of bound protons. If there are too many protons, their mutual Coulombic repulsion limits their mobility [71].

\section{Differences in the Collisionally Activated Dissociation Mechanisms of Proteins and Peptides}

Intermediate charge states [72] of proteins were found to dissociate extensively at intermediate declustering potentials via multiple dissociation channels including b-ion derived internal fragments not commonly observed with peptides (Figure 4 and Figure 5). In addition, intermediate protein charge states dissociated via the canonical Pro, Ile/Leu/Lys, and Asp/Glu channel cleavages. Compared with high charge states, which give rise to predominantly Pro-mediated cleavage, and low charges states, which give rise to predominantly Asp/Glu-mediated cleavage, intermediate charge states represent the "perfect storm," combining all of these plus the many non-canonical cleavages described below (Figure 5a). The mechanisms leading to the extensive fragmentation of intermediate charge states are explained in detail below, illustrated in Figure 4, and summarized in Figure 5.

We observed non-canonical cleavages both $\mathrm{C}$ - and $\mathrm{N}$ - terminal to Gly, Lys, and Gln; N-terminal to Ser, Tyr, Ile, and Leu; and C-terminal to Pro (Pro37-Pro38). The presence of Pro-Xaa cleavage is typically not observed for peptides, especially when the amino acid residues are Pro-Pro [15, 73]. As far as we are aware, the prominent cleavages C-terminal to Asn, and less extensively to Gln, and N-terminal to Ile and Leu, are novel, whereas enhanced, mobile proton-mediated cleavages N-terminal to Gly, Ser, Lys, and Tyr, and charge localized cleavage C-terminal to Lys were previously described [15]. Although the mechanisms of cleavages N-terminal to Gln, Ile, Lys, and Leu have not been described, we revisited a large scale peptide CAD [13] study and were surprised to find these cleavages occur frequently, and are among the few mechanisms that retain charge on b-ions. For example, using unbiased clustering, the Wysocki group determined that peptide fragmentation has four major pathways: X-P, I/L/V-X, $\mathrm{D} / \mathrm{E}-\mathrm{X}$, and b\&y [13]. Many of the non-canonical Nterminal fragments observed here are also observed within what Wysocki termed b\&y cluster, which is comprised of missed tryptic cleavages with arginine close to the N-terminus of the peptide. This behavior observed for missed cleavages harmonizes nicely with the present results, as intact proteins have a less ordered distribution of basic residue locations. Distinct fragmentation has been further highlighted with a $\mathrm{MS}^{2}$ data-mining scheme for Pro, Asp, Lys, and Ile of peptides [13].

These uncommon N-terminal cleavages observed here (e.g., X-Gly, Lys, Gln, Ser, Tyr, Ile, and Leu) in turn led to the novel $b$-ion derived internal fragments that are unique to the intermediate charge states of proteins. In contrast, typical C-terminal cleavages (e.g., I/L/V-X and $\mathrm{D} / \mathrm{E}-\mathrm{X})$, which retain charge on the $\mathrm{y}$-ion, often lead to neutral internal fragments and smaller y ions. Over $90 \%$ of the observed internal fragments had

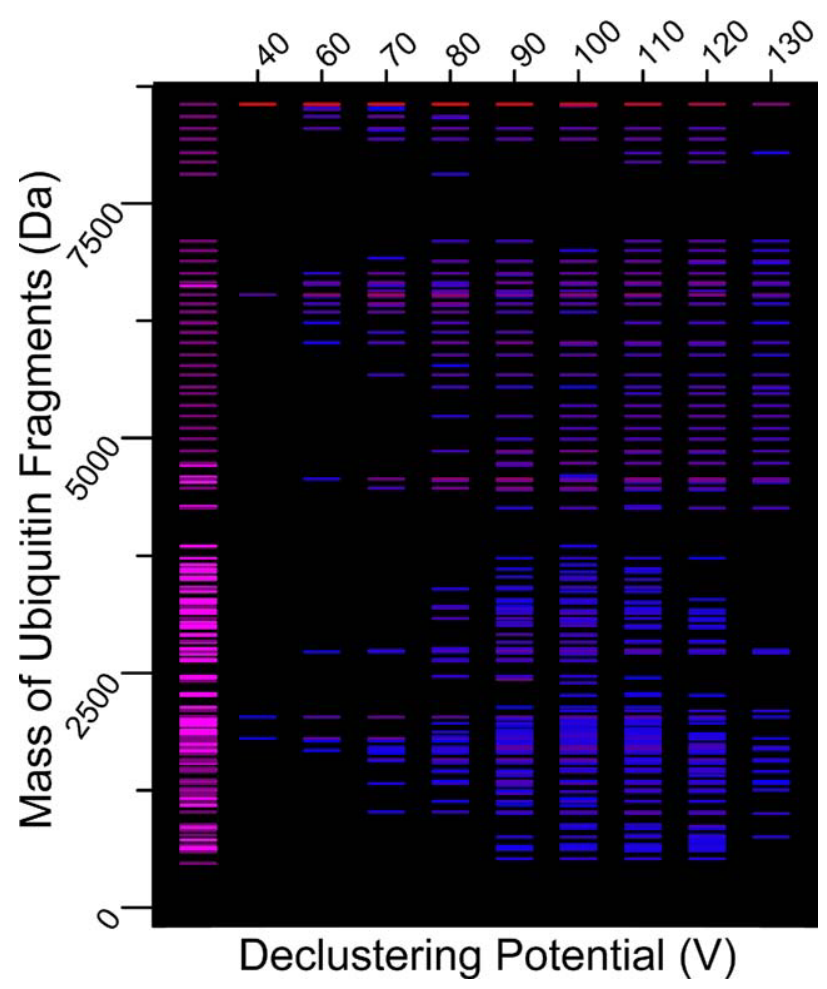

Figure 3. Ubiquitin fragments (in mass domain) as a function of declustering potential $\left(240{ }^{\circ} \mathrm{C}\right.$ capillary temperature). Increased declustering potential resulted in low-energy CAD fragmentation, with cleavage at the amide bond yielding mainly b- and y-type ions. Far left column shows all fragments observed in the mass domain: a-, b-, or y-type (purple), along with all observed internal fragments (magenta). Declustering potentials at 40-130 V (remaining columns) show all locations of fragmentation. The color intensity of the fragments is such that red represents a more intense fragment, while blue represents a less intense fragment, with the color gradient from red to blue on a log scale. 


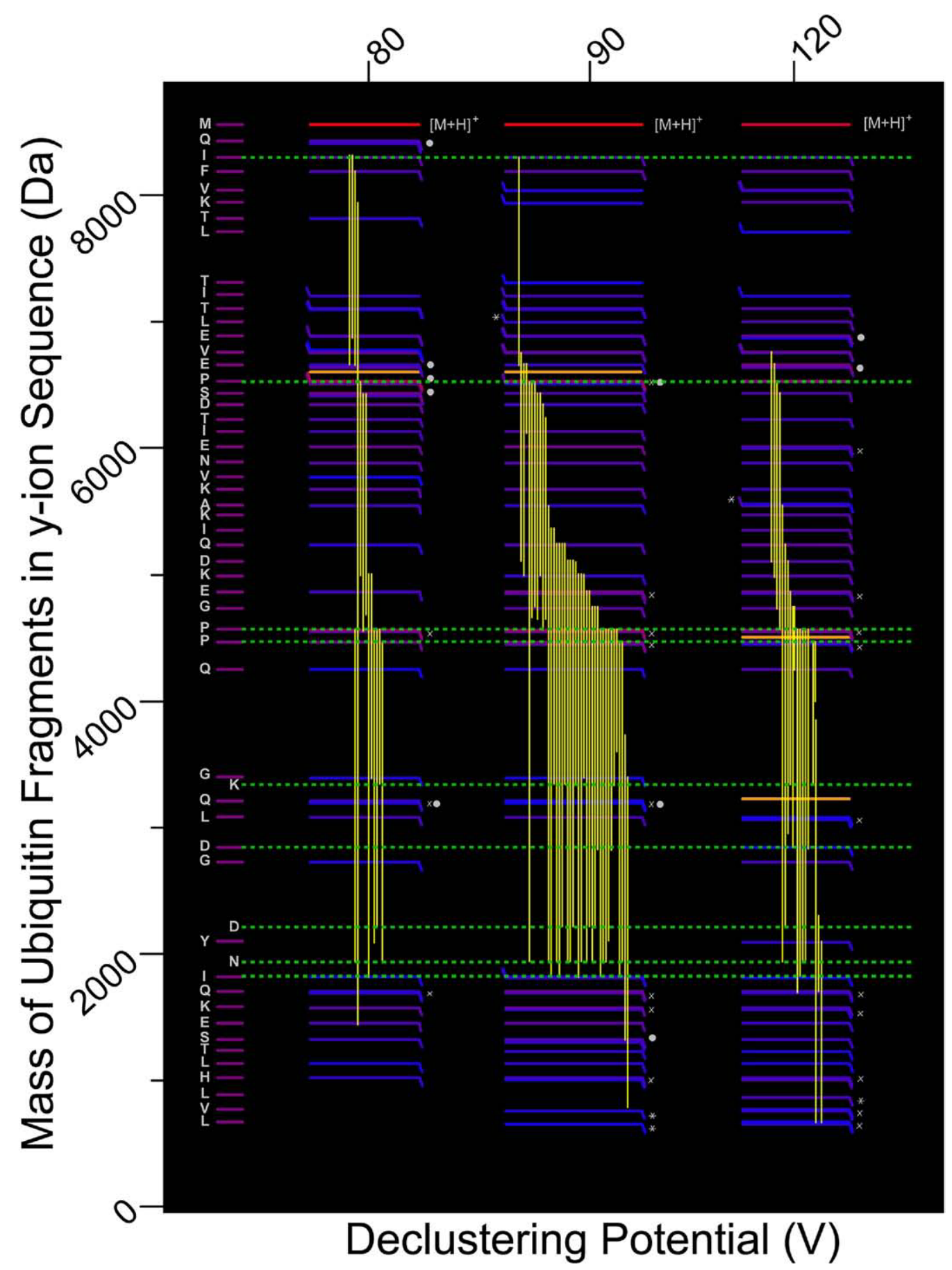

Figure 4. 2D-Gel view of representative funnel-skimmer dissociation fragmentation pathways. Horizontal lines indicate the position where fragmentation occurred, and attached vertical lines indicate the type of ion, with $(\longrightarrow$ ) indicating y-ion and ( $\downarrow-$ ) indicating b-ion. Line color indicates the relative intensity, with blue representing the lowest intensity and red the highest. The amino acid sequence for ubiquitin is depicted in purple on the far left. Yellow vertical bars depict internal fragments in the mass domain from their specified points of fragmentation. Green horizontal bars indicate the major fragmentation pathways. An asterisk at the end of an a-, b-, or y-ion fragment line indicates a loss of $\mathrm{NH}_{3}$. $(\mathrm{X})$ indicates an $\mathrm{a} / \mathrm{b} / \mathrm{y}$ ion present and $\mathrm{a} / \mathrm{b} / \mathrm{y}$ ion with a loss of $\mathrm{NH}_{3}$. (Filled circle) indicates ions in the form of $a / b / y$ ion and $a / b / y$ with a loss of $\mathrm{H}_{2} \mathrm{O}$. Note: interestingly, all fragments were identified except those that appear as the four orange horizontal bars; these were significant, multiply charged, unassigned fragments or protein species that were present based on their mass, not y-ion sequence. 


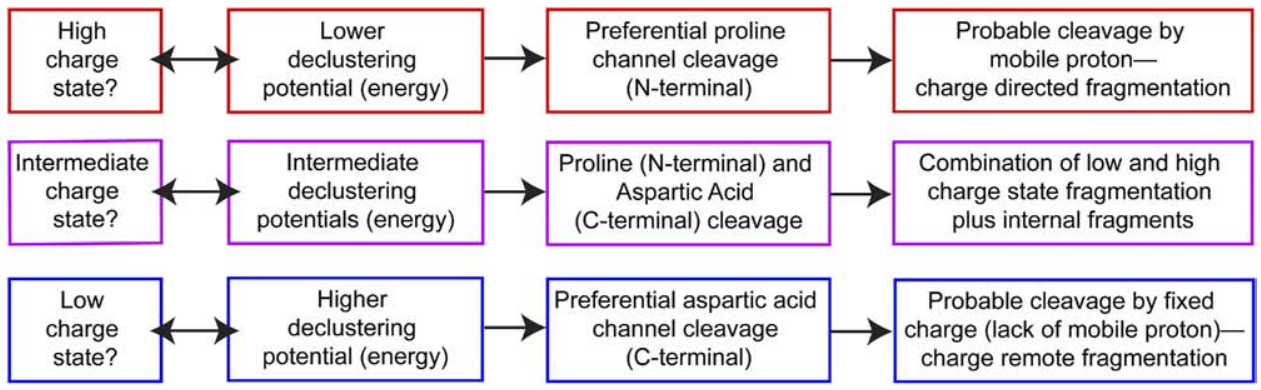

(a)

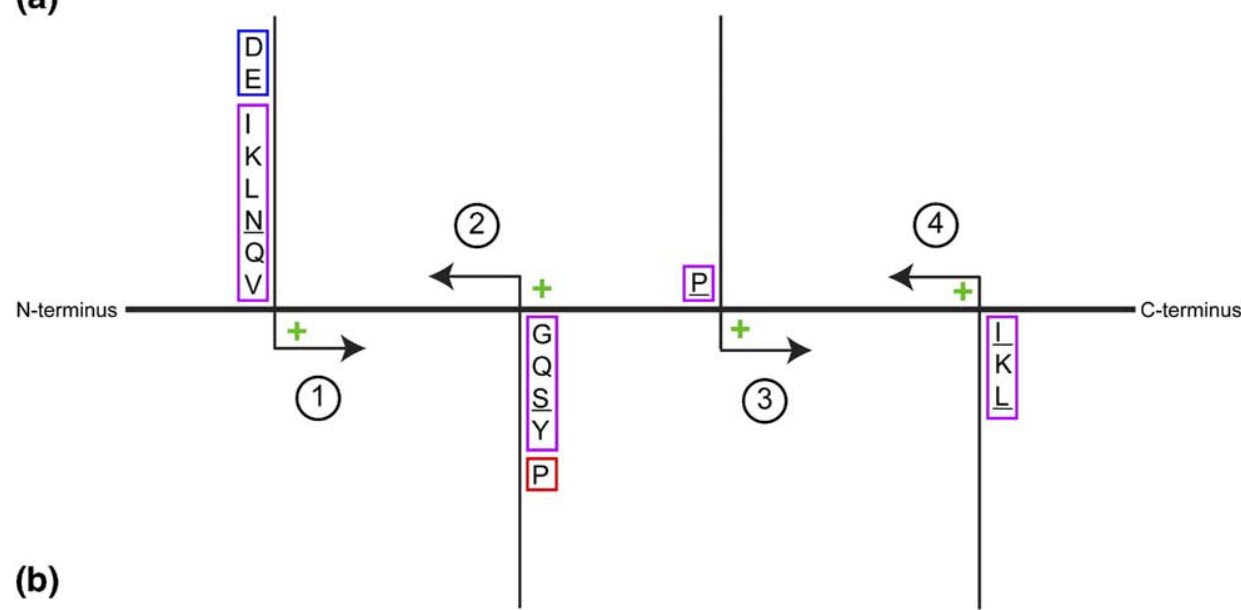

Figure 5. Funnel-skimmer dissociation mechanisms for primary and secondary fragmentation. (a) Summary of charge-state dependence of the fragmentation mechanism. (b) All dominant N- and C-terminal cleavage patterns [underlined amino acids indicate cleavages less common in peptides, red, magenta, and blue boxes correspond to high, intermediate, and low declustering potentials, respectively. Cleavage pattern 1 involved C-terminal cleavage at D, E, I, K, L, N, Q, and V. Cleavage pattern 2 occurred N-terminal to G, Q, S, Y, and P. Cleavage pattern 3 involved C-terminal to P cleavage, which typically is not observed in peptides. In cleavage patterns $\mathbf{1 - 3}$, charge (green + ) is retained on the y-ion. Cleavage pattern 4 involved novel cleavage $\mathrm{N}$-terminal to the amino acids I, K, and $\mathrm{L}$ with the charge being retained on the b-ions; leading to b-ion derived internal fragments.

charges of $1+$ or $2+$ (the remaining were $3+$ or $4+$ ). Indeed, the relatively low charge states of peptides combined with the sequestration of charge at C-terminal basic residues could explain why internal fragment ions are not a prominent feature of peptide CAD. A corollary of our work is that internal fragments should be more predominant in the peptide CAD cluster termed b\&y, and potentially should be accounted for in bottom-up scoring algorithms. Additionally, we observed "steps" in the internal fragmentation pattern that can be visualized in Figure 4 and Figure S9, which led to extensive sequence coverage. These steps can be explained by the fragmentation pathway $b_{x} \rightarrow b_{x-1}$ $\left(b_{x} \rightarrow a_{x} \rightarrow b_{x-1}\right)$ and / or $b_{x} \rightarrow a_{x}$ [12].

Interest in protein intermediate charge states, which give rise to a surprisingly high number of fragments ions (Figure 3) [37], led to ion mobility, proton transfer, blackbody infrared radiative dissociation [74], and hydrogen/deuterium exchange studies [40, 71, 75-77]. These studies demonstrated that intermediate charge states exist in the largest conformational ensemble [39], and are consistent with there being a relationship between collision cross section, conformation, and charge state. However, an open question remains as to whether the enhanced fragmentation of intermediate charge states (Figures 2, 3) results from the larger conformational ensemble [39, 78] or enhanced proton mobility (e.g., there are no mobile protons until the charge state exceeds the number of Arg residues [5], but once there are too many protons, their mutual Coulombic repulsion limits their mobility). In light of evidence that similar fragmentation results from multiple conformations [40], it is more likely that proton mobility is the major player.

\section{Further Increasing Internal Energy Via Capillary Temperature Had Little Effect Upon Protein Dissociation}

A previous study determined that protein fragmentation increased as temperature increased from 38 to $100{ }^{\circ} \mathrm{C}$, accompanied by fewer cleavages leading to $\mathrm{y}$-type ions and increased cleavages leading to internal fragments [39]. This process of activating proteins for CAD using a heated capillary was termed prefolding 
dissociation [39]. Our goal was to maximize the extent of fragmentation. Therefore, we began at the maximum previously characterized temperature and tested even higher temperatures. At the capillary temperatures tested $\left(100,150,200\right.$, and $\left.240{ }^{\circ} \mathrm{C}\right)$, similar fragmentation occurred, indicating that the maximum degree of protein unfolding was achieved by $100{ }^{\circ} \mathrm{C}$, and that a wide range of capillary temperatures can be tolerated. The interested reader is directed to the Supplemental Information for further discussions on the effect of capillary temperature.

\section{Methods for Improving Intact Protein Characterization}

Accounting for dominant internal fragmentation would improve automated protein identification and characterization. The Mascot-TD (previously known as "Big Mascot") score distribution as a function of declustering potential is shown in Figure 6. We modified the Mascot-TD instrument definition to include a-type and internal fragments (see the Experimental section). Paradoxically, the lowest Mascot scores corresponded to the intermediate declustering potentials that yielded the most fragments, indicating that the Mascot-TD scoring algorithm (and perhaps other top-down search algorithms) needs to be recalibrated for intact protein analysis. Specifically, despite a $30 \%$ increase in the total

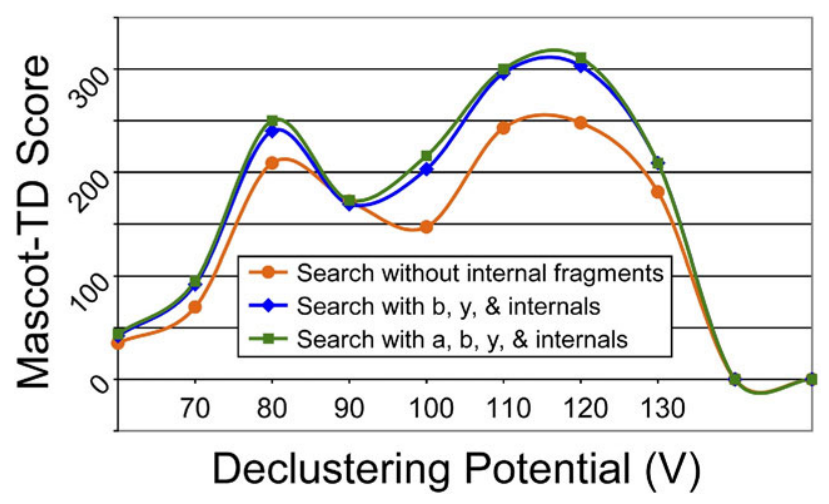

Figure 6. Voltage and search parameter dependence of Mascot-TD score for ubiquitin funnel-skimmer dissociation fragmentation $\left(240{ }^{\circ} \mathrm{C}\right.$ capillary temperature). Internal fragment searches were incorporated as new user-defined instrument definitions to Mascot-TD to help assign the proportion of unmatched ions within the raw data. The same bimodal distribution resulted for ubiquitin searches at all temperatures without internal fragments searched with scores at maxima of $80 \mathrm{~V}$ and $120 \mathrm{~V}$ declustering voltages (see Figure S3e). Orange circle: default search (b- and y-fragments with neutral losses; no internal fragment ions searched). Parameters used for searching data include non-internally calibrated data (using $\mathrm{MS}^{2}$ masses), no internal fragments searched, $0.1 \%$ peptide tolerance, and $0.3 \mathrm{Da}$ fragment mass tolerance. Blue diamond: score results from internally calibrated data, searched without a-type fragments (b, y, and yb-internal fragments), $0.1 \%$ peptide mass tolerance, and $0.05 \mathrm{Da}$ fragment mass tolerance. Green square: score results from internally calibrated data, searched with $\mathrm{a}, \mathrm{b}, \mathrm{y}$, and ya- and yb-internal fragments, $0.1 \%$ peptide mass tolerance and 0.05 Da fragment mass tolerance. number of fragments observed from 80 to $90 \mathrm{~V}$ declustering potentials, the Mascot score decreased by $72 \%$. This was due to the Mascot algorithm [53] having been calibrated using peptide CAD, and as a result not weighting internal fragments. This, combined with the doubling of the percentage of internal fragments between 80 to $90 \mathrm{~V}$ (14 internals/64 total, 41 internals/91 total, respectively), resulted in a lower overall score despite better sequence coverage. Thus, a shortcoming of the current Mascot scoring algorithm, as well as the popular ProSight PTM algorithm [21], is that internal fragment ions do not increase the confidence score to the same extent that $b$ - and $y$-ions do. A zoomed-in view of the internal fragments, grouping similar starting and ending point together, can be found in the Figure S9.

\section{Electronics for Automating Optimized In-Source CAD}

We developed electronics that toggled between the declustering potentials required for intact protein analysis and dissociation. Specifically, automated electronic switching of the declustering potential occurred at every scan to provide a spectrum acquired at a voltage that gave intact mass $(40 \mathrm{~V})$, followed by spectrum acquired at a voltage that dissociated proteins $(>80 \mathrm{~V})$. This automation allowed facile cleavage for identifying and characterizing proteins on a LC timescale, a feat that cannot currently be accomplished using ECD.

\section{Using the Source Region as a Low-Resolution Mass Spectrometer by Modulating of the Declustering Potential}

This application identified declustering potentials that selectively fragment different charge states, allowing the source region to be used as the first stage of a low-resolution tandem mass spectrometer, and facilitating pseudo-MS ${ }^{3}$ of product ions from selected precursor charge states. For example, fragment ions from a declustering potential of $50 \mathrm{~V}$ were derived from the highest charge states, and based upon our analysis above, are likely to be derived from N-terminal proline cleavage.

\section{Conclusions}

We report a comprehensive analysis of funnel-skimmer dissociation with FT-ICR MS instrumentation using ubiquitin, myoglobin, and BSA. The mass spectrometric experiments described here demonstrate that funnelskimmer dissociation is a method capable of producing extensive fragmentation on a LC timescale. Generally, a declustering potential of $100 \mathrm{~V}$ allowed for confident protein identification over a diverse protein molecular weight range (8-67 kDa). Typically, the higher chargestate ions favored fragmentation at proline and adjacent 
residues (at lower voltages), while cleavages C-terminal to aspartic acid and glutamic acid were more prevalent for lower charge-states (at higher voltages). Intermediate charge-states' fragments were a sum of both fragmentation channels plus a higher percentages of internal fragments, leading to the greatest sequence coverage, with novel fragmentation observed N-terminal to Ile, Leu, and Ser, and C-terminal to Asn and Pro. Proper weighting of internal fragments in scoring algorithms will lead to better experimental design and interpretation of results. We developed electronics that can alternate between the ideal declustering potentials for yielding intact protein mass $(40 \mathrm{~V})$ and protein fragmentation $(>80 \mathrm{~V})$, which automated this in-source dissociation methodology. We also demonstrated a general method for using the source region as a low-resolution mass spectrometer, and provided experimental guidelines for tailoring protein fragmentation channels by selectively fragmenting different charge-states (e.g., cleavage at proline versus aspartic acid). Both of these improvements of top-down mass spectrometry afforded facile cleavage of a variety of proteins on a LC timescale.

\section{Acknowledgments}

The authors acknowledge that the results described were supported by funding from the National Institute of Health Award no. R01NS065263 of Neurological Disorders and Stroke. The authors thank all Agar laboratory members for thoughtful discussions, and Clyde Abbott at Bruker Daltonics Inc. for helping with the modified source electronics.

\section{Appendix A Supplementary Material}

Supplementary material associated with this article may be found in the online version at doi:10.1016/ j.jasms.2010.02.009.

\section{References}

1. Fenn, J. B.; Mann, M.; Meng, C. K.; Wong, S. F.; Whitehouse, C. M. Electrospray Ionization for Mass Spectrometry of Large Biomolecules. Science 1989, 246, 64-71.

2. Karas, M.; Hillenkamp, F. Laser Desorption Ionization of Proteins with Molecular Masses Exceeding 10,000 Daltons. Anal. Chem. 1988, 60(20), 2299-2301.

3. Kelleher, N. L.; Lin, H. Y.; Valaskovic, G. A.; Aaserud, D. J.; Fridriksson, E. K.; McLafferty, F. W. Top Down Versus Bottom Up Protein Characterization by Tandem High-Resolution Mass Spectrometry. J. Am. Chem. Soc. 1999, 121(4), 806-812.

4. Karabacak, N. M.; Li, L.; Tiwari, A.; Hayward, L. J.; Hong, P.; Easterling, M. L.; Agar, J. N. Sensitive and Specific Identification of Wild-Type and Variant Proteins from 8 to 669 kDa Using Top-Down Mass Spectrometry. Mol. Cell. Proteom. 2009, 8(4), 846-856.

5. Wysocki, V. H.; Tsaprailis, G.; Smith, L. L.; Breci, L. A. Mobile and Localized Protons: A Framework for Understanding Peptide Dissociation. J. Mass Spectrom. 2000, 35, 1399-1406.

6. Dongré, A. R.; Jones, J. L.; Somogyi,. Á.; Wysocki, V. H. Influence of Peptide Composition, Gas-Phase Basicity and Chemical Modification on Fragmentation Efficiency: Evidence for the Mobile Proton Model. J. Am. Chem. Soc. 1996, 118, 8365-8374

7. Cox, K. A.; Gaskell, S. J.; Morris, M.; Whiting, A. Role of the Site of Protonation in the Low-Energy Decompositions of Gas-Phase Peptide Ions. J. Am. Soc. Mass Spectrom. 1996, 7(6), 522-531.

8. Harrison, A. G.; Yalcin, T. Proton Mobility in Protonated Amino Acids and Peptides. Int. J. Mass Spectrom. Ion Processes 1997, 165, 339-347.

9. Harrison, A. G. The Gas-Phase Basicities and Proton Affinities of Amino Acids and Peptides. Mass Spectrom. Rev. 1997, 16(4), 201-217.
10. Summerfield, S. G.; Gaskell, S. J. Fragmentation Efficiencies of Peptide Ions Following Low Energy Collisional Activation. Int. J. Mass Spectrom. Ion Processes 1997, 165/166, 509-521.

11. Tang, X. J.; Thibault, P.; Boyd, R. K. Fragmentation Reactions of Multiply-Protonated Peptides and Implications for Sequencing by Tandem Mass Spectrometry with Low-Energy Collision-Induced Dissociation. Anal. Chem. 1993, 65(20), 2824-2834.

12. Paizs, B.; Suhai, S. Fragmentation Pathways of Protonated Peptides. Mass Spectrom. Rev. 2005, 24(4), 508-548.

13. Huang, Y.; Tseng, G. C.; Yuan, S.; Pasa-Tolic, L.; Lipton, M. S.; Smith, R. D.; Wysocki, V. H. A Data-Mining Scheme for Identifying Peptide Structural Motifs Responsible for Different MS/MS Fragmentation Intensity Patterns. J. Proteome Res. 2008, 7, 70-79.

14. Tabb, D. L.; Smith, L. L.; Breci, L. A.; Wysocki, V. H.; Lin, D.; Yates, J. R. I. Statistical Characterization of Ion Trap Tandem Mass Spectra from Doubly Charged Tryptic Peptides. Anal. Chem. 2003, 75, 1155-1163.

15. Kapp, E. A.; Schutz, F.; Reid, G. E.; Eddes, J. S.; Moritz, R. L.; O'Hair, R. A. J.; Speed, T. P.; Simpson, R. J. Mining a Tandem Mass Spectrometry Database to Determine the Trends and Global Factors Influencing Peptide Fragmentation. Anal. Chem. 2003, 75(22), 6251-6264.

16. Tabb, D. L.; Huang, Y.; Wysocki, V. H.; Yates, J. R. Influence of Basic Residue Content on Fragment Ion Peak Intensities in Low-Energy Collision-Induced Dissociation Spectra of Peptides. Anal. Chem. 2004 76(5), 1243-1248

17. Tsaprailis, G.; Nair, H.; Somogyi, A.; Wysocki, V. H.; Zhong, W.; Futrell, J. H.; Summerfield, S. G.; Gaskell, S. J. Influence of Secondary Structure on the Fragmentation of Protonated Peptides. J. Am. Chem. Soc. 1999, 121, 5142-5154.

18. Cheng, C.; Gross, M. L. Applications and Mechanisms of ChargeRemote Fragmentation. Mass Spectrom. Rev. 2000, 19(6), 398-420.

19. Buré, C.; Gobert, W.; Lelièvre, D.; Delmas, A. In-Source Fragmentation of Peptide Aldehydes and Acetals: Influence of Peptide Length and Charge State. J. Mass Spectrom. 2001, 36(10), 1149-1155.

20. Nair, H.; Wysocki, V. H. Are Peptides Without Basic Residues Protonated Primarily at the Amino Terminus? Int. J. Mass Spectrom. Ion Processes 1998, 174(1-3), 95-100.

21. LeDuc, R. D.; Taylor, G. K.; Kim, Y. B.; Januszyk, T. E.; Bynum, L. H. Sola, J. V.; Garavelli, J. S.; Kelleher, N. L. ProSight PTM: An Integrated Environment for Protein Identification and Characterization by TopDown Mass Spectrometry. Nucleic Acids Res. 2004, 32 (Web Server issue), W340-345.

22. Senko, M. W.; Speir, J. P.; McLafferty, F. W. Collisional Activation of Large Multiply Charged Ions Using Fourier Transform Mass Spectrometry. Anal. Chem. 1994, 66(18), 2801-2808.

23. Gauthier, J. W.; Trautman, T. R.; Jacobson, D. B. Sustained OffResonance Irradiation for Collision-Activated Dissociation Involving Fourier Transform Mass Spectrometry. Collision-Activated Dissociation Technique that Emulates Infrared Multiphoton Dissociation. Anal. Chim. Acta 1991, 246(1), 211-225.

24. Little, D. P.; Speir, J. P.; Senko, M. W.; O'Connor, P. B.; McLafferty, F. W. Infrared Multiphoton Dissociation of Large Multiply Charged Ions for Biomolecule Sequencing. Anal. Chem. 1994, 66(18), 2809-2815.

25. Zubarev, R. A.; Kelleher, N. L.; McLafferty, F. W. Electron Capture Dissociation of Multiply Charged Protein Cations. A Nonergodic Process. J. Am. Chem. Soc. 1998, 120(13), 3265-3266.

26. Syka, J. E. P.; Coon, J. J.; Schroeder, M. J.; Shabanowitz, J.; Hunt, D. F. Peptide and Protein Sequence Analysis by Electron Transfer Dissociation Mass Spectrometry. Proc. Natl. Acad. Sci. U.S.A. 2004, 101(26), 9528-9533.

27. Han, X.; Jin, M.; Breuker, K.; McLafferty, F. W. Extending Top-Down Mass Spectrometry to Proteins with Masses Greater than 200 Kilodaltons. Science 2006, 314(5796), 109-112.

28. Loo, J. A.; Udseth, H. R.; Smith, R. D.; Futrell, J. H. Collisional Effects on the Charge Distribution of Ions from Large Molecules Formed by Electrospray-Ionization Mass Spectrometry. Rapid Commun. Mass Spectrom. 1988, 2(10), 207-210.

29. Shaffer, S. A.; Prior, D. C.; Anderson, G. A.; Udseth, H. R.; Smith, R. D. An Ion Funnel Interface for Improved Ion Focusing and Sensitivity Using Electrospray Ionization Mass Spectrometry. Anal. Chem. 1998, 70(19), 4111-4119.

30. Shaffer, S. A. Tang, K. Anderson, G. A.; Prior, D. C.; Udseth, H. R. Smith, R. D. A Novel Ion Funnel for Focusing Ions at Elevated Pressure Using Electrospray Ionization Mass Spectrometry. Rapid Commun. Mass Spectrom. 1997, 11(16), 1813-1817.

31. Kaplan, D. A.; Hartmer, R.; Speir, J. P.; Stoermer, C.; Gumerov, D. Easterling, M. L.; Brekenfeld, A.; Kim, T.; Laukien, F.; Park, M. A Electron Transfer Dissociation in the Hexapole Collision Cell of a Hybrid Quadrupole-Hexapole Fourier Transform Ion Cyclotron Resonance Mass Spectrometer. Rapid Commun. Mass Spectrom. 2008, 22(3), 271-278.

32. Gorshkov, M. V.; Masselon, C. D.; Nikolaev, E. N.; Udseth, H. R. Pasa-Tolic, L.; Smith, R. D. Considerations for Electron Capture Dissociation Efficiency in FTICR Mass Spectrometry. Int. J. Mass Spectrom. 2004, 234(1-3), 131-136.

33. Patrie, S. M.; Ferguson, J. T.; Robinson, D. E.; Whipple, D.; Rother, M.; Metcalf, W. W.; Kelleher, N. L. Top Down Mass Spectrometry of $<60-\mathrm{kDa}$ Proteins from Methanosarcina acetivorans Using Quadrupole FTMS with Automated Octopole Collisionally Activated Dissociation. Mol. Cell. Proteom. 2006, 5(1), 14-25. 
34. Forbes, A. J.; Patrie, S. M.; Taylor, G. K.; Kim, Y.-B.; Jiang, L.; Kelleher, N. L. Targeted Analysis and Discovery of Post-Translational Modifications in Proteins from Methanogenic archaea by Top-Down MS. Proc. Natl. Acad. Sci. U.S.A. 2004, 101(9), 2678-2683.

35. Engel, B. J.; Pan, P.; Reid, G. E.; Wells, J. M.; McLuckey, S. A. Charge State Dependent Fragmentation of Gaseous Protein Ions in a Quadrupole Ion Trap: Bovine ferri-, ferro-, and apo-Cytochrome c. Int. J. Mass Spectrom. 2002, 219(1), 171-187.

36. Newton, K. A.; Chrisman, P. A.; Reid, G. E.; Wells, J. M.; McLuckey, S. A. Gaseous Apomyoglobin Ion Dissociation in a Quadrupole Ion Trap: $[\mathrm{M}+2 \mathrm{H}]^{2+}-[\mathrm{M}+21 \mathrm{H}]^{21+}$. Int. J. Mass Spectrom. 2001, 212(1-3), 359-376.

37. Reid, G. E.; Wu, J.; Chrisman, P. A.; Wells, J. M.; McLuckey, S. A. Charge-State-Dependent Sequence Analysis of Protonated Ubiquitin Ions via Ion Trap Tandem Mass Spectrometry. Anal. Chem. 2001, 73(14), 3274-3281.

38. Stephenson, J. L.; McLuckey, S. A.; Reid, G. E.; Wells, J. M.; Bundy, J. L. Ion/Ion Chemistry as a Top-Down Approach for Protein Analysis. Curr. Opin. Biotechnol. 2002, 13(1), 57-64.

39. Zhai, H.; Han, X.; Breuker, K.; McLafferty, F. W. Consecutive Ion Activation for Top Down Mass Spectrometry: Improved Protein Sequencing by Nozzle-Skimmer Dissociation. Anal. Chem. 2005, 77(18), 5777-5784.

40. Badman, E. R.; Hoaglund-Hyzer, C. S.; Clemmer, D. E. Dissociation of Different Conformations of Ubiquitin Ions. J. Am. Soc. Mass Spectrom. 2002, 13(6), 719-723.

41. Koeniger, S. L.; Clemmer, D. E. Resolution and Structural Transitions of Elongated States of Ubiquitin. J. Am. Soc. Mass Spectrom. 2007, 18(2), 322-331.

42. Zubarev, R. Protein Primary Structure Using Orthogonal Fragmentation Techniques in Fourier Transform Mass Spectrometry. Exp. Rev. Proteom. 2006, 3(2), 251-261.

43. Johnson, J. R.; Meng, F.; Forbes, A. J.; Cargile, B. J.; Kelleher, N. L. Fourier-Transform Mass Spectrometry for Automated Fragmentation and Identification of 5-20 kDa Proteins in Mixtures. Electrophoresis 2002, 23(18), 3217-3223.

44. Bogdanov, B.; Smith, R. D. Proteomics by FTICR Mass Spectrometry: Top Down and Bottom Up. Mass Spectrom. Rev. 2005, 24(2), 168-200.

45. Riba Garcia, I.; Giles, K.; Bateman, R. H.; Gaskell, S. J. Studies of Peptide a- and b-Type Fragment Ions Using Stable Isotope Labeling and Integrated Ion Mobility/Tandem Mass Spectrometry. J. Am. Soc. Mass Spectrom. 2008, 19(12), 1781-1787.

46. Allen, J. M.; Racine, A. H.; Berman, A. M.; Johnson, J. S.; Bythell, B. J.; Paizs, B.; Glish, G. L. Why Are a3 Ions Rarely Observed? J. Am. Soc. Mass Spectrom. 2008, 19(12), 1764-1770.

47. Bythell, B. J.; Somogyi, Á.; Paizs, B. What is the Structure of b2 Ions Generated from Doubly Protonated Tryptic Peptides? J. Am. Soc. Mass Spectrom. 2009, 20(4), 618-624.

48. Oomens, J.; Young, S.; Molesworth, S.; van Stipdonk, M. Spectroscopic Evidence for an Oxazolone Structure of the b2 Fragment Ion from Protonated Tri-Alanine. J. Am. Soc. Mass Spectrom. 2009, 20(2), 334-339.

49. Slepukhina, I.; Duelcks, T.; Schiebel, H.-M.; Gabel, D. Fragmentation of $\mathrm{B}_{12} \mathrm{H}_{11} \mathrm{~S}-\mathrm{R}(2-)$ in Electrospray Mass Spectrometry. J. Org. Chem. 2005, 690(11), 2796-2801.

50. Roepstorff, P.; Fohlman, J. Proposal for a Common Nomenclature for Sequence Ions in Mass Spectra of Peptides. Biomed. Mass Spectrom. 1984, 11(11), 601.

51. Biemann, K. Contributions of Mass Spectrometry to Peptide and Protein Structure. Biomed. Environ. Mass Spectrom. 1988, 16, 99-111.

52. Koster, C. Mass spectrometry method for accurate mass determination of unknown ions. 2001

53. Perkins, D. N.; Pappin, D. J. C.; Creasy, D. M.; Cottrell, J. S. ProbabilityBased Protein Identification by Searching Sequence Databases Using Mass Spectrometry Data. Electrophoresis 1999, 20(18), 3551-3567.

54. We found no significant effect in score when re-searching the data with the higher upper mass limit at $110 \mathrm{kDa}$ for Mascot-TD so we chose to present all data searched in the same manner; however, we note that in theory, predicting y-ions requires an intact protein mass so it was worthwhile to re-search with the updated software.

55. Thomson, B. A. Declustering and Fragmentation of Protein Ions from an Electrospray Ion Source. J. Am. Soc. Mass Spectrom. 1997, 8(10), 10531058.

56. Loo, J. A.; Edmonds, C. G.; Udseth, H. R.; Smith, R. D. Collisional Activation and Dissociation of Large Multiply Charged Proteins Produced by Electrospray Ionization. Anal. Chim. Acta 1990, 241(2), 167173.
57. Loo, J. A.; Edmonds, C. G.; Smith, R. D. Primary Sequence Information from Intact Proteins by Electrospray Ionization Tandem Mass Spectrometry. Science 1990, 248(4952), 201-204.

58. Loo, J. A.; Edmonds, C. G.; Smith, R. D. Tandem Mass Spectrometry of Very Large Molecules: Serum Albumin Sequence Information from Multiply Charged Ions Formed by Electrospray Ionization. Anal. Chem. 1991, 63(21), 2488-2499.

59. Loo, J. A.; Edmonds, C. G.; Smith, R. D. Tandem Mass Spectrometry of Very Large Molecules. 2. Dissociation of Multiply Charged ProlineContaining Proteins from Electrospray Ionization. Anal. Chem. 1993, 65(4), 425-438

60. Busman, M.; Rockwood, A. L.; Smith, R. D. Activation Energies for Gas-Phase Dissociations of Multiply Charged Ions from Electrospray Ionization Mass Spectrometry. J. Phys. Chem. 1992, 96(6), 2397-2400.

61. Ashton, D. S.; Beddell, C. R.; Cooper, D. J.; Green, B. N.; Oliver, R. W. A Mechanism of Production of Ions in Electrospray Mass Spectrometry. Org. Mass Spectrom. 1993, 28(6), 721-728.

62. Schaaff, T. G.; Cargile, B. J.; Stephenson, J. L.; McLuckey, S. A. Ion Trap Collisional Activation of the $(\mathrm{M}+2 \mathrm{H})^{2+}-(\mathrm{M}+17 \mathrm{H})^{17+}$ Ions of Human Hemoglobin B-Chain. Anal. Chem. 2000, 72(5), 899-907.

63. Gardner, M. W.; Brodbelt, J. S. Impact of Proline and Aspartic Acid Residues on the Dissociation of Intermolecularly Crosslinked Peptides. J. Am. Soc. Mass Spectrom. 2008, 19(3), 344-357.

64. Stephenson, J. L.; McLuckey, S. A. Simplification of Product Ion Spectra Derived from Multiply Charged Parent Ions Via Ion/Ion Chemistry. Anal. Chem. 1998, 70(17), 3533-3544.

65. Yu, W.: Vath, J. E.; Huberty, M. C.: Martin, S. A. Identification of the Facile Gas-Phase Cleavage of the Asp-Pro and Asp-Xxx Peptide Bonds in Matrix-Assisted Laser Desorption Time-of-Flight Mass Spectrometry. Anal. Chem. 1993, 65(21), 3015-3023.

66. Qin, J.; Chait, B. T. Preferential Fragmentation of Protonated Gas-Phase Peptide Ions Adjacent to Acidic Amino Acid Residues. J. Am. Chem. Soc. 1995, 117(19), 5411-5412.

67. Jockusch, R. A.; Schnier, P. D.; Price, W. D.; Strittmatter, E. F.; Demirev, P. A.; Williams, E. R. Effects of Charge State on Fragmentation Pathways, Dynamics, and Activation Energies of Ubiquitin Ions Measured by Blackbody Infrared Radiative Dissociation. Anal. Chem. 1997, 69(6), $1119-1126$

68. Gu, C.; Tsaprailis, G.; Breci, L.; Wysocki, V. H. Selective Gas-phase Cleavage at the Peptide Bond Terminal to Aspartic Acid in FixedCharge Derivatives of Asp-Containing Peptides. Anal. Chem. 2000, 72, 5804-5813.

69. Kruger, N. A.; Zubarev, R. A.; Carpenter, B. K.; Kelleher, N. L.; Horn, D. M.; McLafferty, F. W. Electron Capture Versus Energetic Dissociation of Protein Ions. Int. J. Mass Spectrom. 1999, 182/183, 1-5.

70. Rockwood, A. L.; Busman, M.; Udseth, H. R.; Smith, R. D. Thermally Induced Dissociation of Ions from Electrospray Mass Spectrometry. Rapid Commun. Mass Spectrom. 1991, 5(12), 582-585.

71. Li, J.; Taraszka, J. A.; Counterman, A. E.; Clemmer, D. E. Influence of Solvent Composition and Capillary Temperature on the Conformations of Electrosprayed Ions: Unfolding of Compact Ubiquitin Conformers from Pseudonative and Denatured Solutions. Int. J. Mass Spectrom. 1999, 185/187, 37-47

72. In keeping with the McLuckey group's designation for ubiquitin, high charge states are defined as fully protonated $(+13)$ to +10 , intermediate charge states as $+9,+8$, and +7 , and low charge states as +6 and below.

73. Breci, L. A.; Tabb, D. L.; Yates, J. R.; Wysocki, V. H. Cleavage N-Terminal to Proline: Analysis of a Database of Peptide Tandem Mass Spectra. Anal. Chem. 2003, 75(9), 1963-1971.

74. Gross, D. S.; Zhao, Y.; Williams, E. R. Dissociation of Heme-Globin Complexes by Blackbody Infrared Radiative Dissociation: Molecular Specificity in the Gas Phase? J. Am. Soc. Mass Spectrom. 1997, 8(5), $519-524$.

75. Myung, S.; Badman, E. R.; Lee, Y. J.; Clemmer, D. E. Structural Transitions of Electrosprayed Ubiquitin Ions Stored in an Ion Trap over $10 \mathrm{~ms}$ to 30 s. J. Phys. Chem. A 2002, 106(42), 9976-9982.

76. Valentine, S. J.; Counterman, A. E.; Clemmer, D. E. ConformerDependent Proton-Transfer Reactions of Ubiquitin Ions. J. Am. Soc. Mass Spectrom. 1997, 8(9), 954-961.

77. Freitas, M. A.; Hendrickson, C. L.; Emmett, M. R.; Marshall, A. G. Gas-Phase Bovine Ubiquitin Cation Conformations Resolved by GasPhase Hydrogen/Deuterium Exchange Rate and Extent. Int. J. Mass Spectrom. 1999, 185/187, 565-575.

78. Breuker, K.; Oh, H.; Horn, D. M.; Cerda, B. A.; McLafferty, F. W. Detailed Unfolding and Folding of Gaseous Ubiquitin Ions Characterized by Electron Capture Dissociation. J. Am. Chem. Soc. 2002, 124(22), 6407-6420. 